\title{
Thermal desorption of cryopumped gases from laser treated copper
}

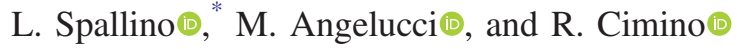 \\ LNF-INFN, Frascati (Roma), Italy
}

(Received 8 April 2020; accepted 11 June 2020; published 22 June 2020)

\begin{abstract}
Recently, laser processing of copper samples has been demonstrated to produce rough surfaces whose nanostructuring ensures unquestionable advantages for electron cloud mitigation in future particle accelerators. The actual application of laser treated surfaces in accelerators implies that this new material is compliant with many issues, going from impedance vacuum properties to many others. A significant experimental effort is therefore ongoing to study and optimize their various properties of interest. Here we analyze their vacuum behavior versus temperature. To this end, we studied thermal programmed desorption from $\mathrm{CO}, \mathrm{CH}_{4}$ and $\mathrm{H}_{2}$ once cryosorbed on laser treated copper substrate and on its flat counterpart. These molecules are typically present in the residual vacuum of any accelerator. The results show that the desorption of such gases from the laser treated substrates occurs in a much broader and higher temperature range with respect to what is observed from the flat substrate. We also show that, at equal doses, treated samples adsorb/desorb significantly more gas than their flat counterpart. These findings can be ascribed to their nanostructured porous morphology. A quantitative analysis is given, allowing to properly estimate fluctuations of the number of molecules during unavoidable temperature variations of the cryogenic vacuum system.
\end{abstract}

DOI: 10.1103/PhysRevAccelBeams.23.063201

\section{INTRODUCTION}

One of the major topics in material science oriented to accelerators $R \& D$ is the search of electron cloud effect (ECE) mitigation strategies [1-6]. ECEs are one serious potential showstopper for ultimate performance of accelerators of positively charged particles. Low energy electrons, generated in accelerator vacuum chambers by photoemission, residual-gas ionization and secondary emission can seriously affect accelerators' operation and performance in a variety of ways. They can induce increases in vacuum pressure, emittance growth, beam instabilities, beam losses, beam lifetime reductions, or additional heat loads on a (cold) chamber wall [5-13]. Such "cloud" electrons will be accelerated by the positive beam, gaining energy and will finally hit the vacuum chamber. There, they will create additional secondary electrons, whose number is governed by the secondary electron yield (SEY) of the wall surface. SEY is defined as the ratio between the number of emitted electrons (also called secondary electrons) to the number of incident electrons (also called primary electrons). When SEY is

\footnotetext{
*luisa.spallino@lnf.infn.it
}

Published by the American Physical Society under the terms of the Creative Commons Attribution 4.0 International license. Further distribution of this work must maintain attribution to the author(s) and the published article's title, journal citation, and DOI. larger than unity, the electron population multiplies with successive bunch passages. This can lead to the formation of an increasingly high density of electrons in the cloud and, hence, to ECE. Therefore, one powerful method to control and overcome such effects is to construct accelerators' vacuum chambers with a low SEY surface, ideally always less than or just around one.

Recently, it has been shown that treating a metallic surface by laser ablation surface engineering (LASE) is extremely successful in reducing SEY below 1 [14,15]. In those pioneering works, Valizadeh et al. showed that LASE gives to the surface a particular morphology formed by different scale structures, micrometrical grooves with coral-like submicron agglomeration of nanospheres. SEY is therefore reduced by a known geometrical effect [2,16-20]. Since LASE "modified" surfaces appeared on the scene, a number of experimental activities have started to validate such surfaces with respect to a number of other requirements. Magnetic properties, vacuum and constructive compatibility, powder production, impedance issues (both geometric and due to surface conductivity) are among the most stringent ones [21]. This wide research activity fits well in a more general approach, necessary when qualifying a material to be used in accelerators. A significant effort, for instance, was done to validate LASE to have surface conductivity within the usually available minimal impedance budget. In respect to the flat substrate, LASE structuring could significantly affect surface conductivity and may have a crucial impact on machine impedance [13,22-26]. A recent study on a specific 
LASE type has shown that its surface resistance depends on the direction of the surface currents relative to the laserengraved groove pattern [27]: it increases by less than $10 \%$ for parallel patterns, but, in the perpendicular case, it is 4-5 times higher than the surface resistance of the flat counterpart. Work is still ongoing, and a certain optimization of the structure seems to be very promising, tailoring morphology to both maximize conductivity and maintain a low SEY $[6,13,25]$.

As laser treatment acts on $\mathrm{Cu}$, which indeed is a vacuum compatible material, one could expect that a morphological modification would preserve this property. On the other hand, it is known that porous materials have an effective surface significantly larger than their flat counterpart. This significant surface increase could certainly influence its vacuum behavior. Moreover, a yet more severe issue arises when considering to use such LASE treated surfaces at cryogenic temperatures $(\mathrm{T})$. It is well known that porous structures efficiently trap surface contaminants, broadening and shifting to higher temperatures the desorption of gases [6,28-36]. Gas ices cryosorbed on flat surfaces desorb, in ultrahigh vacuum, at specific and known temperatures [37-39]. Therefore, the operational temperature of a cryogenic chamber in accelerators is chosen not only for costs and impedance reasons, but also to be far from such desorption temperature onsets [21]. This strategy allows to avoid vacuum transients during cold wall temperature fluctuations. In LHC, for example, such $\mathrm{T}$ oscillations are of the order of $\sim 5 \mathrm{~K}$ and are unavoidable and intrinsic to the complex control network of the cooling loops $[38,39]$. In case of strongly morphologically modified vacuum chamber surfaces held at cryogenic temperature, the evaluation of the saturated vapor pressure curves may not be sufficient to assess the absence of vacuum transient during small temperature fluctuations.

Recently, a specific study on Ar gas cryosorbed on LASE-Cu showed that Ar ice does not desorb from this treated surface in a very limited temperature range (as from flat surfaces) but desorbs in a much larger interval [36]. In that work, Ar was chosen as a prototypical inert gas but it is certainly not representative of any gas species present, as residual gas, in an accelerator's vacuum systems. A more thorough review of typical residual gas desorption kinetics from a representative LASE surface is therefore mandatory. The scope of the present paper is to analyze temperature programmed desorption (TPD) from various coverages of $\mathrm{CO}, \mathrm{CH}_{4}$ and $\mathrm{H}_{2}$ on a prototypical copper treated surface (LASE-Cu). The data here collected are not enough, by themselves, to conclusively address the vacuum compatibility of LASE in cryogenic vacuum. Many more studies will be required also to qualify LASE "dynamic" vacuum behavior. Its study implies addressing LASE electron-, photon- and ion-stimulated desorption (ESD, PSD, ISD). In fact, all such phenomena will govern vacuum during the accelerator's operation and need to be carefully considered and quantified. Once more experimental data are collected, then simulations will be conclusively used to validate the implementation of LASE in accelerators as it is, or if it requires some more refinement and further optimization. Then, the data here presented will be an important input for such vacuum simulation programs. In fact, they can be used to calculate the allowed temperature fluctuation range and the relative amount of gas finally delivered inside the vacuum chambers.

\section{EXPERIMENTAL METHODS}

Experiments were performed in ultrahigh vacuum (UHV) in a $\mu$-metal chamber at the "material science" laboratory of the INF-LNF. The setup has been described elsewhere [5,8,36,40-43].

In brief, UHV conditions are reached by a cryopump, having Ar pumping speed equal to $1200 \mathrm{l} / \mathrm{s}$. The system operates in $p<1 \times 10^{-10}$ mbar after bakeout. The setup allows fast sample transfer in UHV, enabling untreated samples to be inserted directly from air and studied without suffering from thermal stress and modifications due to bakeout. A close cycle He cooled cryogenic manipulator allows to hold the sample at $15<\mathrm{T}<300 \pm 0.2 \mathrm{~K}$.

As schematically shown in Fig. 1(a), the cold manipulator can be moved and rotated to put the sample in different

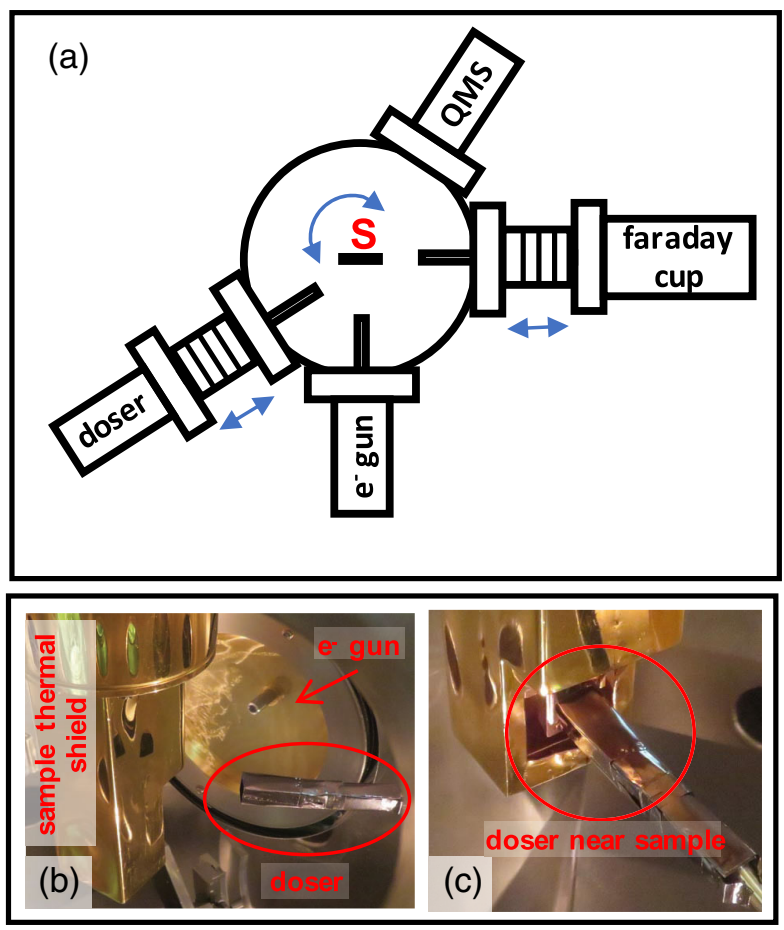

FIG. 1. (a) Schematic representation of the ultrahigh (UHV) vacuum experimental setup. Pictures (b) and (c) show the dosing configurations adopted in this study. Red labels indicate the different components. (b) In the retracted position, the doser is far from the sample (S) and secondary electron yield (SEY) measurements can be performed during gas delivering. (c) Putting the doser very close to the sample allows to reduce the background signal in TPD measurements. 
working configurations. The following operations can be performed: (i) Ice growth. - A gas line is connected to the chamber with a "home-made" gas-dosing system, ending up with an $\sim 8 \times 8 \mathrm{~mm}^{2}$ square (doser) which nearly exactly matches the samples size. This doser has chicanes to obtain a more homogenous gas flow on the sample surface and can be inserted very close (less than $1 \mathrm{~mm}$ ) to the sample surface or retracted away from it. Different positions of the doser are chosen depending on the experiment, as it will be explained in the following sections. In the configuration with the doser far from the sample, as shown in Fig. 1(b), the gas reaches all parts of the cold manipulator and, then, increases the TPD background signal. This effect is effectively mitigated by dosing near the sample, as shown in Fig. 1(c). In this configuration, the gas adsorbed on the cold manipulator is significantly reduced and so is the background signal in the TPD measurements. Of course, when dosing in this configuration, coverages cannot be directly inferred by pressure readings but need to be calibrated. Here we do this preliminary but fundamental operation by studying SEY of $\mathrm{Ar}$ on clean $\mathrm{Cu}$ as a prototypical system. (ii) Secondary electron yield measurements. - As said, SEY is here used as a coverage calibration method. To perform SEY measurements, the sample is irradiated by an electron beam of the order of $0.5 \mathrm{~mm}^{2}$ in the transverse cross-sectional area, emitted by an Omicron electron gun equipped with a standard $\mathrm{LaB}_{6}$ cathode. The gun is placed $\sim 5 \mathrm{~cm}$ from the sample and SEY is performed at normal incidence. SEY is determined by measuring the primary electrons' current, $\mathrm{I}_{p}$, and the sample drain current, $\mathrm{I}_{s}$, independently $[5,8,36,40-43]$. SEY is then given:

$$
\mathrm{SEY}=\frac{I_{p}-I_{s}}{I_{p}}=1-\frac{I_{s}}{I_{p}} .
$$

$\mathrm{I}_{p}$ is measured by a home-made Faraday cup that can be moved in front of the $e^{-}$gun. The Faraday cup collector is positively biased $\left(\mathrm{V}_{s}=75 \mathrm{~V}\right)$ in order to prevent backscattered reemission to vacuum. To confidently measure SEY up to low impinging electron energy (few hundreds of $\mathrm{meV}$ ), a negative bias voltage of $\mathrm{V}_{s}=-75 \mathrm{~V}$ is applied to the sample to determine $\mathrm{I}_{s}$ [42]. For the present investigation, SEY measurements were performed using electron beam currents of a few $\mathrm{nA}$ to induce minimal electron desorption or conditioning during data acquisition [41]. (iii) Thermal programmed desorption measurements.TPD investigations are performed by using a quadrupole mass spectrometer (QMS, Hiden, HAL 3F PIC) while heating the sample with a rate of $0.005 \mathrm{~K} / \mathrm{s}$. A resistive heater controlled by a diode is used to increase the temperature. TPD curves are given in molecules (or atoms, in the case of Ar) per unit of geometrical area, which is independently from surface morphology. The conversion from counts (given by QMS) and molecules $\cdot \mathrm{cm}^{-2} \cdot \mathrm{K}^{-1}$ is made possible by assuming that, in the coverage range we consider, when dosing on the polycrystalline flat sample, the sticking coefficient is unity. As shown later, this assumption is very reasonable for $\mathrm{Ar}, \mathrm{CO}$ and $\mathrm{CH}_{4}$. Then, after a calibrated dose of $10 \mathrm{~L}$ of gas on the flat polycrystalline surface $\left(1 \mathrm{~L}=1.33 \times 10^{-6} \mathrm{mbar} \cdot \mathrm{s}\right)$, we have ten monolayers (ML) of gas adsorbed on the surface and the integral of its desorption has been used to calibrate the mass spectrometer. Literature results [44-50] show that, for the gas types here considered, we can assume that $1 \mathrm{ML}$ contains a number of molecules (or atoms) per $\mathrm{cm}^{2}$ of the order $\sim 10^{15} \pm 10 \%$. In this way, when we desorb $10 \mathrm{~L}$ of any gas dosed on the flat surface, the integral of the signal coming from QMS accounts for the desorption of $\sim 10 \times$ $10^{15}$ molecules (or atoms). This calibrates the QMS, independently from the surface morphology. All desorptions are done in well controlled and reproducible conditions and in a regime where the signal read by the QMS is linear with the amount of gas. Pressure has been corrected scaling the gauge readings for the sensitivities to the different gas species. As explained later, only the TPD from $\mathrm{H}_{2}$ cannot be scaled to numbers of molecules and will be given in partial pressure, maintaining only a qualitative value.

\section{MATERIALS}

Two categories of $\mathrm{Cu}$ sample were investigated, having dimension $\sim 8 \times 8 \mathrm{~mm}^{2}$ : a sputtered clean "flat" $\mathrm{Cu}$ substrate and a representative sample from the LASE-Cu material family. (i) poly-Cu. The flat polycrystalline $\mathrm{Cu}$ (poly-Cu) consists of Oxygen-Free High Conductivity (OFHC) copper substrate. It has been atomically cleaned (as proven by SEY) by repeated $\mathrm{Ar}^{+}$sputtering cycles at $\sim 1 \mathrm{keV}$, to have a reproducible surface for calibration and comparison purposes. This cleaning procedure does not alter the surface roughness, which is and stays about $50 \mathrm{~nm}$, as estimated by AFM measurements. (ii) LASE-Cu. The LASE-Cu sample was treated by laser ablation surface engineering by R. Valizadeh et al. at the Science \& Technology Facility Council (STFC). It consists of a OFHC copper sheet, of some hundreds of microns, colaminated on stainless steel and then laser treated [15]. The sample surface morphology has been probed at LNF facility by scanning electron microscopy (SEM), using a SNE-3200M tabletop SEM. As shown in Fig. 2, LASE-Cu surface is characterized by an inhomogeneous submicrometric highly porous network having a coral-like structure of agglomerated nanoparticles. SEM images confirm that the sample here analyzed is morphologically congruent to other similar studied LASE samples $[14,15,22,36]$ and has to be considered a relevant reference sample for the category.

In this study, TPD of 10, 25 and 50 Langmuir (L) of $\mathrm{CO}$ and $\mathrm{CH}_{4}$ are reported, together with the TPD of $100 \mathrm{~L}$ of $\mathrm{H}_{2}$. Ultrapure gases ( $\left.\geq 99.9999 \%\right)$ from low pressure 


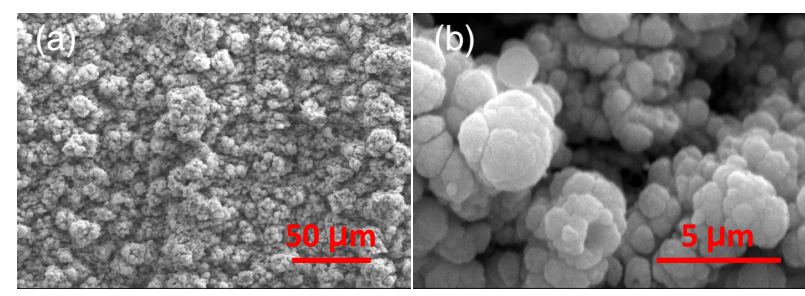

FIG. 2. (a) and (b): Scanning electron microscopy (SEM) micrographs, acquired with different magnifications, of the LASE sample under investigation [36].

(12 bar) bottles were all delivered through a UHV compatible gas line (base pressure $p \leq 1 \times 10^{-7}$ mbar) connected to the measuring system via a calibrated leak valve. During gas dosing far from the sample, as in the configuration shown in Fig. 1(b), the pressure in the measuring system was always maintained $p_{\text {far }} \leq 5 \times 10^{-8}$ mbar; while, when dosing close to the sample, as in the configuration shown in Fig. 1(c), the pressure in the measuring system was always maintained $p_{\text {close }} \leq 2 \times 10^{-9}$ mbar. The desired doses were then chosen varying the "exposure time" to the surfaces and were defined with an accuracy of $\pm 10 \%$, as given by the accuracy of pressure readings.

In all cases, the specific gas was delivered on the substrate held at $15 \mathrm{~K}$. The dose region under consideration allows us to investigate, for the flat sample, the so-called "multilayer regime," where the interaction between the substrate surface and the adsorbed gas can be ignored. Moreover, this is the typical gas coverage range expected to occur in long exposures to residual vacuum in an accelerator cryogenic environment [39].

\section{A. Determination of coverage for the flat poly-Cu sample}

When dosing near the sample, as in the configuration shown in Fig. 1(c), coverages cannot be given by "pressure" $\times$ "exposure time" since the gas impingement rate on the substrate is not the same as the one on the pressure gauge and mass spectrometer. The SEY technique has been identified as a precious method to calibrate coverage in the regime explored in this work. For this purpose, an inert gas like Ar was dosed far from sample [as in Fig. 1(b)] on the flat substrate (poly-Cu) and SEY was measured versus coverage. In this configuration, the gas dose measured by the pressure gauge is the same as the one "seen" by the surface. SEY of a flat $\mathrm{Cu}$ surface is strongly sensitive to the presence of Ar overlayer onto the surface and its value clearly and steeply depends on its thickness [36,51].

Figure 3(a) shows SEY curves as a function of Ar dose on the flat poly-Cu sample, from bare $(0 \mathrm{~L})$ to $135 \mathrm{~L}$. In this coverage interval, SEY clearly increases with coverage. Reporting the SEY value at $900 \mathrm{eV}$ as a function of Ar dose [Fig. 3(b)], a clear linear trend is observed, and can be fitted by the following function:
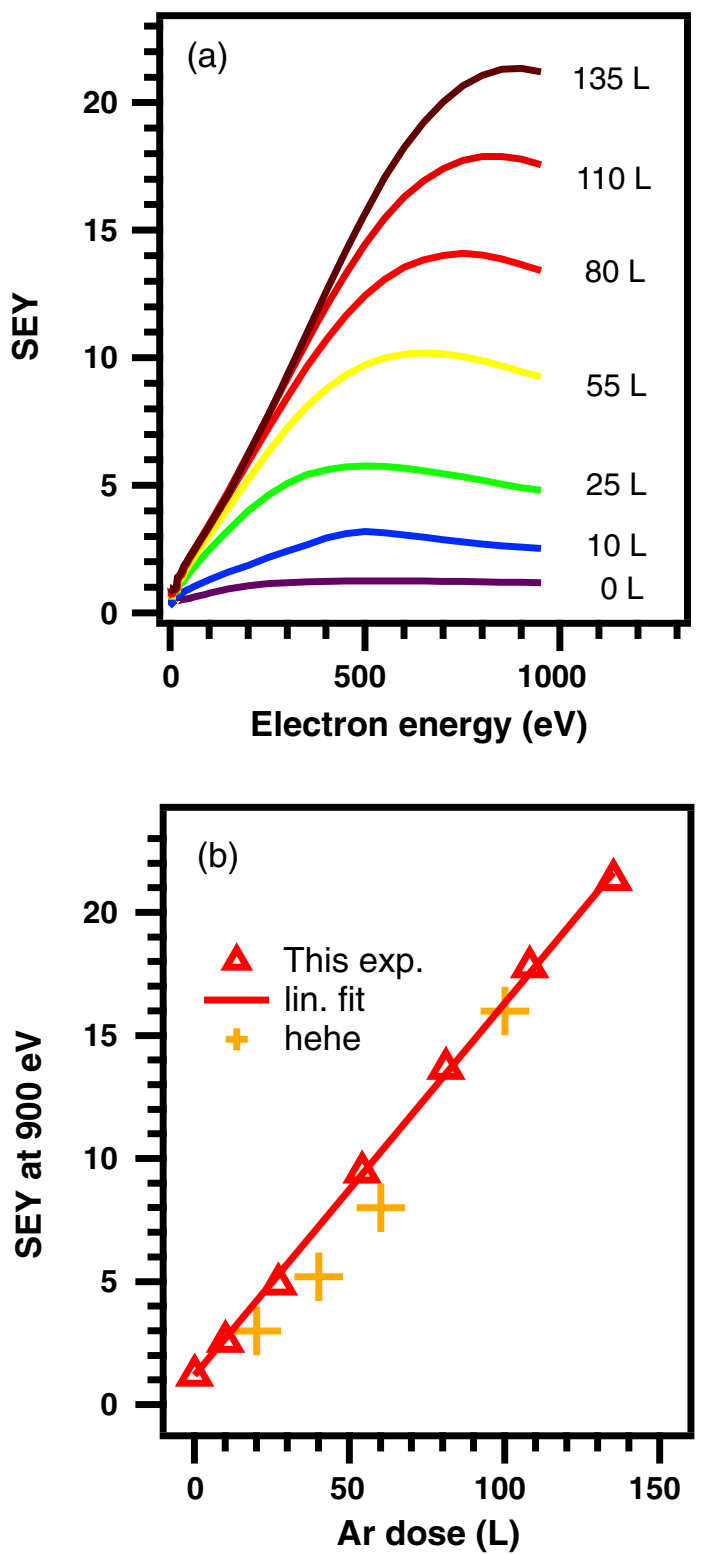

FIG. 3. (a) Secondary electron yield (SEY) curves measured for increasing $\mathrm{Ar}$ doses on the flat poly-Cu sample. (b) Triangles indicate the trend obtained from the curves in panel (a), plotting the SEY value at $900 \mathrm{eV}$ as a function of Ar dose. Cross symbols refer to data extrapolated from data by Cazaux et al. [51]. The solid line, in panel (b), indicates the best fit curve of the experimental points obtained using Eq. (2).

$$
\delta^{900}=\delta_{\mathrm{Cu}}^{900}+a \cdot D
$$

where $\delta^{900}$ is the $\mathrm{SEY}$ value at $900 \mathrm{eV}, \delta_{\mathrm{Cu}}^{900}=1.2$ is the SEY measured at $900 \mathrm{eV}$ from the bare poly-Cu sample, D indicates the Ar dose, in Langumir, and $a=0.1513 \pm$ 0.0012 is the coefficient obtained from fit. As shown by the cross symbols in Fig. 3(b), within an uncertainty of $\pm 20 \%$, the trend observed in this dosing range is in agreement with the SEY value at $900 \mathrm{eV}$ extrapolated from the curves 
reported by Cazaux et al. [51]. Without entering into the physics governing SEY of noble gas layers condensed on a $\mathrm{Cu}$ substrate, it is clear that we can use this experimental evidence to calibrate our doses when the dosing is performed near the sample.

After dosing Ar close to the flat poly-Cu sample, as in the configuration shown in Fig. 1(c), SEY directly gives the thickness of the Ar ice condensed on the substrate with an accuracy of $\pm 20 \%$. Therefore, we can establish the proportionality between the pressures measured by the QMS and vacuum gauge, and the effective pressure seen by the sample. It is reasonable to assume that this ratio is independent from the used gas and, therefore, we can calibrate all doses with an accuracy of $\pm 30 \%$. Even if we lose some accuracy in the dose estimation, this method gives us the possibility to perform all the TPD experiments with the desired low background signal.

The equivalence between the dose (given in Langmuir) and the ice thickness (given in monolayers) cannot be used for LASE-Cu. Clearly, the thickness of an Ar layer is significantly different on the porous and on the flat substrates, even for nominally equal doses. Moreover, the assumptions that the Ar pressure seen by the flat surface is homogeneous in all the porous fractals and that the sticking coefficient, for the LASE-Cu, is homogeneously 1 are certainly not valid. Therefore, the number of molecules (or atoms) adsorbed onto the LASE-Cu may depend on the actual sample nanostructure and coverages on LASE-Cu result ill defined. Here, the Langmuir unit is used as the only common variable for both cases.

\section{RESULTS}

Figure 4 shows the TPD curves obtained monitoring in UHV the desorption of 10,25 , and $50 \mathrm{~L}$ of $\mathrm{CH}_{4}$ dosed on the poly-Cu (a) and LASE-Cu (b) sample. The curves are normalized as discussed in the experimental section. A sharp peak at $\mathrm{T} \sim 35-39 \mathrm{~K}$ characterizes the desorption curves of $\mathrm{CH}_{4}$ on the poly-Cu sample (a). The peaks have a full width at half maximum FWHM $\sim 4 \mathrm{~K}$. In agreement with previous literature findings [52,53], this single peak corresponds to the desorption of a condensed thick $\mathrm{CH}_{4}$ layer. When $\mathrm{CH}_{4}$ is dosed on the LASE-Cu substrate, the TPD curves are characterized by a dose-dependent broad profiles. Together with the sharp $\mathrm{CH}_{4}$ desorption peak at $\mathrm{T} \sim 35-39 \mathrm{~K}$, a wide component appears in a temperature range between $\sim 50$ and $\sim 100 \mathrm{~K}$ with a maximum around $70-75 \mathrm{~K}$, depending on the dose.

In Fig. 5 the TPD curves of 10, 25, and $50 \mathrm{~L}$ of $\mathrm{CO}$ are reported. Panel (a) is referred to $\mathrm{CO}$ on the poly- $\mathrm{Cu}$ substrate, panel (b) to $\mathrm{CO}$ on the LASE-Cu sample. The desorption curve of $\mathrm{CO}$ on poly-Cu consists of a sharp peak at $\mathrm{T} \sim 28-30 \mathrm{~K}$, with FWHM $\sim 4 \mathrm{~K}$. This peak is characteristic of the desorption of a condensed thick $\mathrm{CO}$ layer [52-54] and its temperature is determined by the Van der Waals interaction energies between CO molecules [37,55].
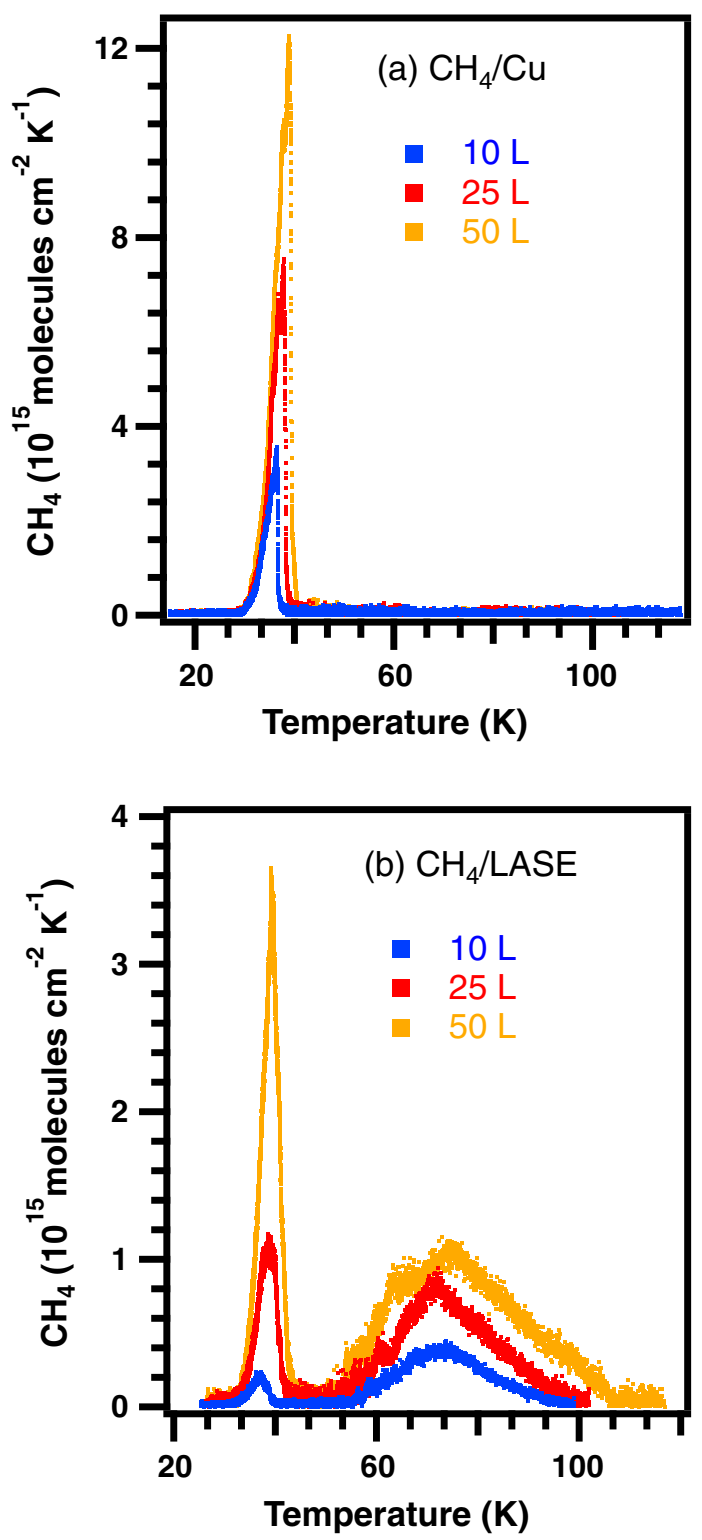

FIG. 4. TPD curves as a function of $\mathrm{CH}_{4}$ dose on flat poly- $\mathrm{Cu}$ substrate (a); LASE-Cu sample (b).

The TPD curves of CO on LASE-Cu (b) are characterized by composite features, extending from $\sim 50$ to $140 \mathrm{~K}$ and with maxima between $\sim 70$ and $\sim 110 \mathrm{~K}$. Moreover, after 25 and $50 \mathrm{~L}$, the $\mathrm{CO}$ desorption at $\mathrm{T} \sim 28-30 \mathrm{~K}$ is also observed.

Qualitative TPD curves obtained after dosing $100 \mathrm{~L}$ of $\mathrm{H}_{2}$ on poly-Cu and LASE-Cu samples are reported in Fig. 6. No signal is observed if $\mathrm{H}_{2}$ is delivered on the flat substrate. This is expected since at the temperature and pressures at which we dose ( $T=15 \mathrm{~K}$ and $p \sim 10^{-9}$ mbar $)$ no $\mathrm{H}_{2}$ multilayer is supposed to stick on the sample [56]. Only below $3 \mathrm{~K} \mathrm{H}_{2}$ can be found as a multilayer on a solid surface in UHV $[37,56]$. On the other hand, a desorption profile is detected if $\mathrm{H}_{2}$ is dosed on the porous substrate. This consists in two broad contributions having peaks at 

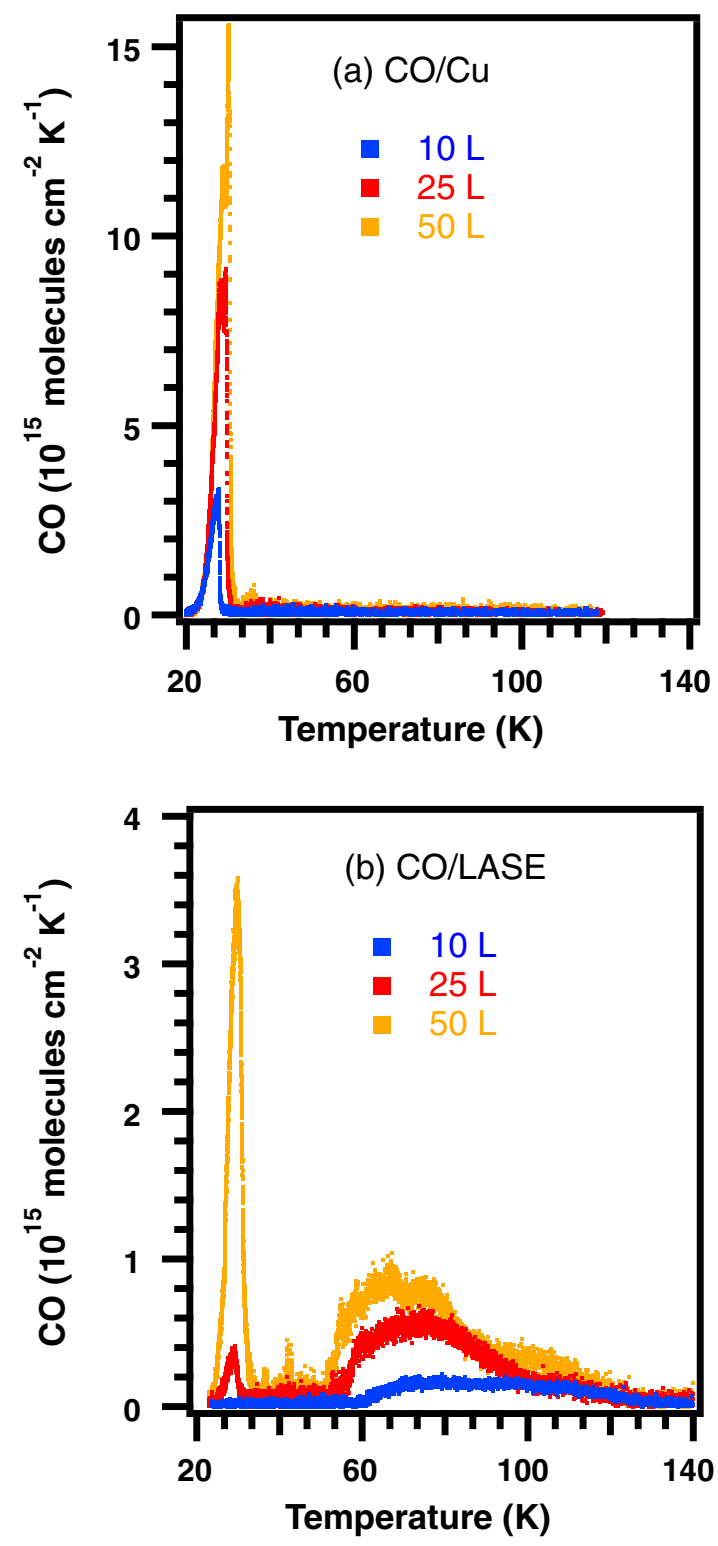

FIG. 5. TPD curves as a function of $\mathrm{CO}$ dose on flat poly- $\mathrm{Cu}$ substrate (a); LASE-Cu sample (b).

$\sim 60$ and $\sim 90 \mathrm{~K}$, both having FWHM $\sim 10 \mathrm{~K}$. Figure 6 dramatically shows how the notions extracted from vapor pressure data [37] can be misleading when the substrate is porous in nature.

\section{DISCUSSION}

Thermal desorption properties of $\mathrm{CO}, \mathrm{CH}_{4}$ and $\mathrm{H}_{2}$ dosed on two $\mathrm{Cu}$ surfaces of different morphology have been reported. For completeness, the data will be integrated with the ones on $\mathrm{Ar}$ [36]. A common trend can be highlighted for all species. This can be summarized as follows: (i) When measuring TPD from a flat surface, a sharp peak is observed for all species, except $\mathrm{H}_{2}$, since there, the sample temperature in our UHV setup was not low enough to grant

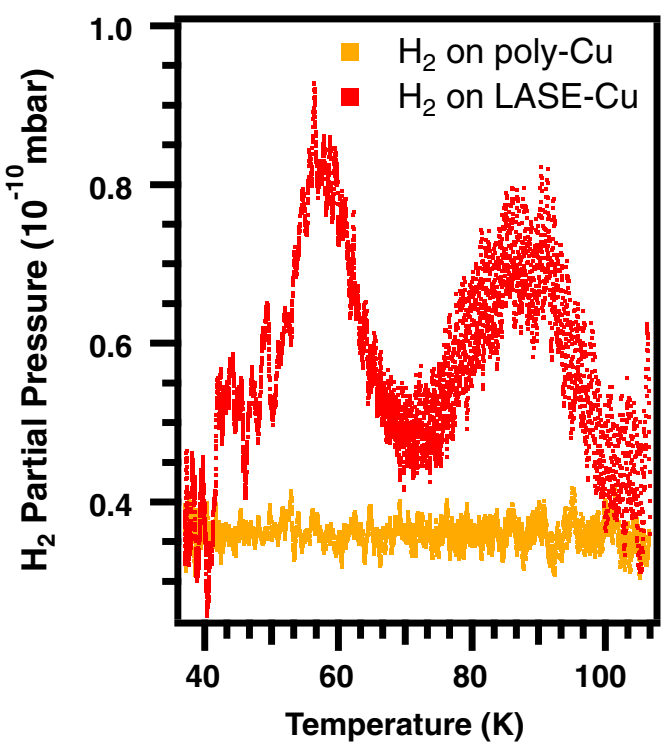

FIG. 6. TPD curves from $100 \mathrm{~L}_{\text {of }} \mathrm{H}_{2}$ dosed on the flat poly- $\mathrm{Cu}$ and on LASE-Cu samples.

any molecular sticking [37]. This single peak corresponds to the desorption of a condensed thick layer of the specific gas and its temperature is determined by the weak Van der Waals interaction energies between molecules (or atoms, in case of Ar) [37,55]. (ii) The desorption curves of all gases and coverages from the LASE-Cu surface are characterized by broad profiles. The range of temperatures and the shape depend on specific gas and on dose.

Laser treatment not only increases the area accessible to atomic/molecular species but its nanostructured morphology determines a local increase of the adsorption energy for atoms/molecules in correspondence of undercoordinated sites and defects [57-60]. The desorption of the atoms/ molecules close to defected surfaces and/or trapped in the pores of the LASE-Cu surface is shifted to higher temperature. Therefore, also $\mathrm{H}_{2}$ desorption from LASE-Cu can be observed, even if during the dosing procedure the substrate is at a temperature higher than the one known to be required for any condensation in UHV. This result can be compared with a previous study by Moulard et al. [59]. Their anodized Al porous samples were kept between 3.7 and $7 \mathrm{~K}$ during gas dosing. The temperature range in which we observe the $\mathrm{H}_{2}$ desorption from LASE-Cu substrate is different from the one reported by [59] for $\mathrm{H}_{2}$ on porous $\mathrm{Al}$. This can be ascribed to the different chemistry and microporosity of the two samples.

A striking feature when looking at some curves [for instance in Fig. 5(b) for the two lowest CO coverages] is that the peak at higher temperature has more intensity than the multilayer peak at lower temperature. Intuitively one could think that the higher binding energy sites are in the pores and more difficult to reach in the conditions of reduced mobility at $15 \mathrm{~K}$. A detailed discussion on the driving forces governing the observed desorption behaviors 


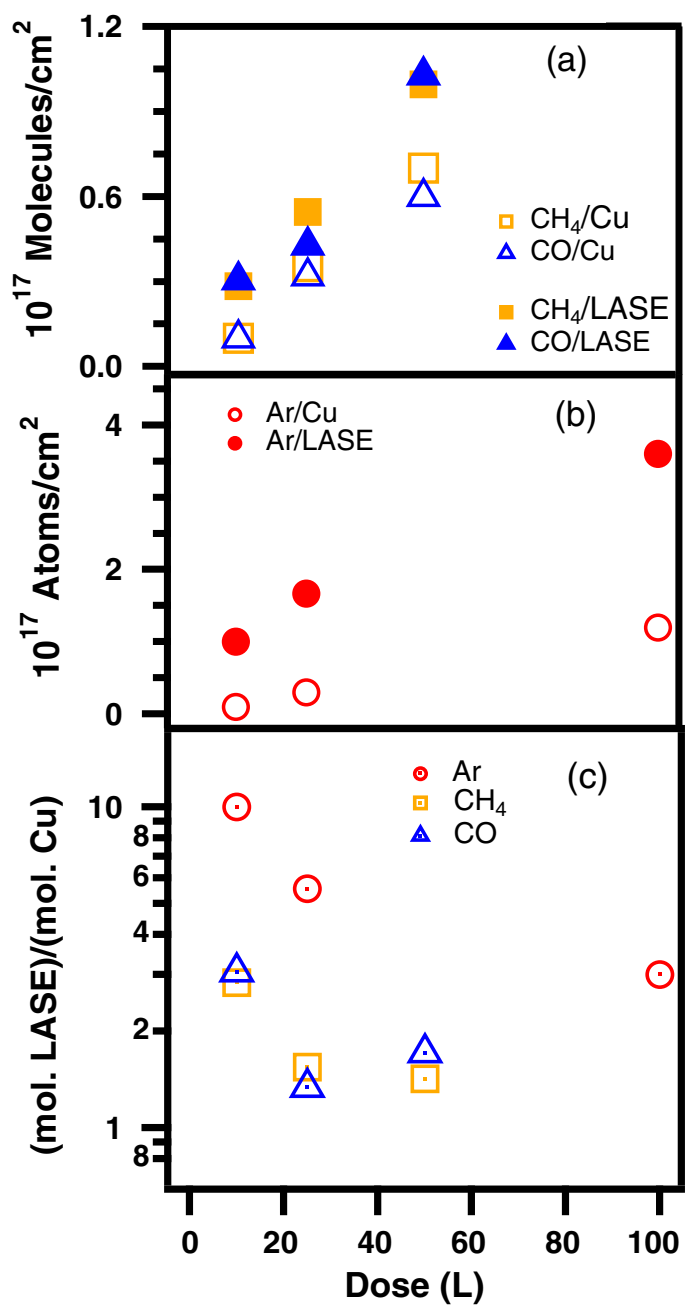

FIG. 7. (a) Total number of $\mathrm{CH}_{4}$ and $\mathrm{CO}$ molecules $/ \mathrm{cm}^{2}$ desorbed from poly- $\mathrm{Cu}$ flat surface (open symbol) and from LASE-Cu (full symbols) as a function of gas dose. (b) Total number of $\mathrm{Ar}$ atoms $/ \mathrm{cm}^{2}$ desorbed from poly- $\mathrm{Cu}$ flat surface (full symbol) and from LASE-Cu (open symbols) as a function of gas dose [36]. (c) Ratio between the number of $\mathrm{CH}_{4}$ and $\mathrm{CO}$ molecules (Ar atoms) desorbed from LASE-Cu and poly- $\mathrm{Cu}$ sample as a function of gas dose.

is outside the scope of the present paper. A possible model describing our observation can be found in Paldor et al. [33]. Moreover, the peculiarities of the TPD curves here reported could depend on the actual LASE sample under examination, more than being a general property of all LASE materials.

A more detailed analysis can be performed by looking at the total gas desorbed from the surfaces during the thermal cycles. The results are shown in Fig. 7 and are obtained integrating each curve given in Figs. 5 and 4. Data extracted from Ar TPD curves shown in a previous publication [36] are also included in Fig. 7(b). The areas are given in molecules (or atoms) per $\mathrm{cm}^{2}$, following the normalization procedure described in the experimental section. In Figs. 7(a) and 7(b), it can be observed that, for the flat sample (open symbols), the area under the sharp desorption peak perfectly scales with the gas dose, confirming that, in the coverage range under consideration, the sticking coefficient stays constant and close to one. On the contrary, the integrated gas quantity released by the LASE-Cu during TPD still increases with dose but not proportionally to it. A kind of saturation process is possibly taking place, so that one could speculate that, for this surface, we have a coverage dependent sticking probability. This assumption could be qualitatively justified by considering that, at the beginning, some adsorption sites of the morphological complex LASE surface have a very high sticking coefficient (close to one) and then, with coverage, it gets reduced.

In Fig. 7(c), for each gas species and as a function of dose, we report the ratio between the integral of the TPD curves coming from the gas dosed on the two different substrates. Some considerations can be done by looking at the figure: (i) Independently from the gas species, the integral of the TPD obtained from gas on the porous substrate is always higher than the one related to the same gas on the flat surface; it can be 10 times higher (as for $10 \mathrm{~L}$ of Ar) down to 1.5 times (as for $50 \mathrm{~L}$ of $\mathrm{CH}_{4}$ ), but the uptake (and consequent release) at equal doses is constantly higher from the LASE-Cu. (ii) Independently from the gas species, the ratio is not constant in all dose ranges under consideration and it is higher at the lower dose; extrapolation to even lower dose regimes, that are outside the scope this study, could show much higher ratio values. (iii) The results are quantitatively about the same for $\mathrm{CH}_{4}$ and $\mathrm{CO}$. The Ar trend appears qualitatively similar but the ratio assumes higher values.

In the investigated coverage region, the ratio of gas delivered from LASE-Cu versus the one delivered from the flat surface is significantly far from the one expected considering the actual surface aspect ratio that can be derived from Fig. 2. This could be justified by the occurrence of two different and concomitant effects. To start with, we notice here that the LASE-Cu is an "as-received" surface and, most likely, various air contaminants are already saturating most of its deep lying adsorption sites. This can significantly reduce the actual surface available for further adsorption. Also, the lowest dose we deliver $(10 \mathrm{~L})$ is actually high enough to contribute in reducing the number of effective adsorption sites. This is why the area ratio decreases with increasing coverages and why Ar, being an inert monoatomic species, gives rise to a higher ratio, still decreasing with dose. Two extreme conditions can be described: a perfectly clean porous material and a material where all the pores and fractal structures are filled by gas ice. In the first case, low dose TPD ratio will be proportional to the area ratio between the flat and porous surface. In the second case, gas adsorption will take place as on the flat surface. From the data we conclude that the investigated porous surface has a surface capacity much below the surface aspect ratio and that the 
history of the LASE surface may play a role in determining its desorption (and adsorption) kinetics. This is in agreement with data by Paldor et al. [33] obtained by studying the desorption characteristics of Xe from a flat and a highly porous Si substrate. Also in that study, the initial Xe uptake, close to be proportional to surface ratio, decreases on increasing the gas dose due to the progressive occupation of all available adsorption sites in the pores. At this stage and concurrently, the contribution deriving from the thick ice, which is forming on the topmost surface, starts to become significant and independent from the substrate morphology.

\section{CONCLUSION}

LASE surfaces have been proposed to be used in accelerators to successfully mitigate electron cloud related effects. Much work is ongoing to validate their properties to be compliant to the many demanding requirements a novel material have to pass before being proposed and used. Among others, vacuum properties have to be carefully analyzed to grant that their intrinsic porous nature, and hence their significantly augmented active surface, does not negatively influence their vacuum behavior. A first step in this direction is presented in this work, where we review the temperature dependent desorption properties of typical gasses $\left(\mathrm{CO}, \mathrm{CH}_{4}\right.$ and $\mathrm{H}_{2}$ ) composing residual vacuum in accelerators and in quantities expected to be delivered to the surface during long term operation. On the flat $\mathrm{Cu}$ surface, studied here as a reference, thermal desorptions occur in a quite limited $(\leq 4 \mathrm{~K})$ temperature window and at a specific temperature which depends on the adsorbate specie. On the contrary, we show here that cryosorbed gases desorb from a LASE surface in a much wider temperature interval. Such large $\mathrm{T}$ window can extend by more than 50 to $100 \mathrm{~K}$, depends on the actual coverage and, most probably, on the detailed surface nanostructure. Then, what is here reported for the species under investigation has to be expected to occur for all gas. Also, the gas uptake (and therefore released) from a LASE surface is significantly more than on the flat surface, even if not as much as could be expected just by considering the actual geometrical surface. Varying porosity by varying LASE production parameters may be an efficient way to optimize TPD performances.

More than presenting trends and qualitative analysis, care has been devoted to give quantitative estimates of TPD signals. The data here reported will therefore help vacuum scientists to properly estimate gas quantity fluctuations during unavoidable temperature variations of the cryogenic vacuum system. Simulations will be used to evaluate whether such T dependent gas releases from LASE surfaces are compatible with the machine normal operation or if some additional care should be taken. To finally validate LASE vacuum behavior in accelerators also nonthermal mechanisms, like ion-, electron-, and photo-stimulated desorption, should be carefully studied.

\section{ACKNOWLEDGMENTS}

This work was supported by the "MICA" project funded by INFN Scientific National Committee 5 . We thank R. Larciprete for stimulating discussions, and V. Baglin, P. Chiggiato, R. Kersevan, O. Malyshev, and R. Valizadeh for useful discussions and for the LASE samples. We acknowledge the DAФNE-L team for technical assistance, in particular G. Viviani for the SEM analysis of the LASE samples. L. S. and M. A. acknowledge the support of The European Circular Energy-Frontier Collider Study (EuroCirCol) project (Grant No. 654305).

[1] Y. Suetsugu, K. Kanazawa, K. Shibata, and H. Hisamatsu, Nucl. Instrum. Methods Phys. Res., Sect. A 556, 399 (2006).

[2] M. Pivi, F. King, R. Kirby, T. Raubenheimer, G. Stupakov, and F. Le Pimpec, J. Appl. Phys. 104, 104904 (2008).

[3] Y. Suetsugu, H. Fukuma, L. Wang, M. Pivi, A. Morishige, Y. Suzuki, M. Tsukamoto, and M. Tsuchiya, Nucl. Instrum. Methods Phys. Res., Sect. A 598, 372 (2009).

[4] C. Y. Vallgren, G. Arduini, J. Bauche, S. Calatroni, P. Chiggiato, K. Cornelis, P. C. Pinto, B. Henrist, E. Métral, H. Neupert, G. Rumolo, E. Shaposhnikova, and M. Taborelli, Phys. Rev. ST Accel. Beams 14, 071001 (2011).

[5] R. Cimino and T. Demma, Int. J. Mod. Phys. A 29, 1430023 (2014).

[6] O. Malyshev, Vacuum in Particle Accelerators: Modelling, Design and Operation of Beam Vacuum Systems (Wiley, New York, 2019).

[7] G. Rumolo, A. Z. Ghalam, T. Katsouleas, C. K. Huang, V. K. Decyk, C. Ren, W. B. Mori, F. Zimmermann, and F. Ruggiero, Phys. Rev. ST Accel. Beams 6, 081002 (2003).

[8] R. Cimino, I. Collins, M. Furman, M. Pivi, F. Ruggiero, G. Rumolo, and F. Zimmermann, Phys. Rev. Lett. 93, 014801 (2004).

[9] F. Zimmermann, Phys. Rev. ST Accel. Beams 7, 124801 (2004).

[10] ECLOUD'12: Joint INFN-CERN-EuCARD-AccNet Workshop on Electron-Cloud Effects, edited by R. Cimino, G. Rumolo, and F. Zimmermann (CERN, Geneva, 2013), CERN Yellow Reports: Conference Proceedings.

[11] K. Ohmi, F. Zimmermann, L. Mether, and D. Schulte, CERN Technical Report No. CERN-ACC-2015-285, Geneva, Switzerland, 2015.

[12] G. Rumolo and G. Iadarola, CERN Yellow Reports: School Proceedings 3, 411 (2017).

[13] Proceedings of ECLOUD'18, edited by R. Cimino, G. Rumolo, and F. Zimmermann (CERN, Geneva, 2020), CERN Yellow Reports: Conference Proceedings.

[14] R. Valizadeh, O. B. Malyshev, S. Wang, S. A. Zolotovskaya, W. A. Gillespie, and A. Abdolvand, Appl. Phys. Lett. 105, 231605 (2014). 
[15] R. Valizadeh, O. Malyshev, S. Wang, T. Sian, M. D. Cropper, and N. Sykes, Appl. Surf. Sci. 404, 370 (2017).

[16] A. Krasnov, Vacuum 73, 195 (2004).

[17] V. Nistor, L. A. González, L. Aguilera, I. Montero, L. Galán, U. Wochner, and D. Raboso, Appl. Surf. Sci. 315, 445 (2014).

[18] C. Swanson and I. D. Kaganovich, J. Appl. Phys. 120, 213302 (2016).

[19] M. Ye, D. Wang, and Y. He, J. Appl. Phys. 121, 124901 (2017).

[20] C. Swanson and I. D. Kaganovich, J. Appl. Phys. 122, 043301 (2017).

[21] V. Baglin, P. Lebrun, L. Tavian, and R. van Weelderen, Technical Report No. CERN-ATS-2013-006, CERN, Geneva, 2013.

[22] S. Calatroni, E. G.-T. Valdivieso, H. Neupert, V. Nistor, A. T. P. Fontenla, M. Taborelli, P. Chiggiato, O. Malyshev, R. Valizadeh, S. Wackerow et al., Phys. Rev. Accel. Beams 20, 113201 (2017).

[23] M. Migliorati, E. Belli, and M. Zobov, Phys. Rev. Accel. Beams 21, 041001 (2018).

[24] ICFA Mini-Workshop on Impedances and Beam Instabilities, edited by V. Brancolini, G. Rumolo, M. R. Masullo, and S. Petracca (CERN, Geneva, 2018), CERN Yellow Reports: Conference Proceedings.

[25] ICFA Mini-Workshop on Mitigation of Coherent Beam Instabilities in particle accelerators MCBI 2019, edited by G. Metral, E. Rumolo, and T. Pieloni, CERN Yellow Reports: Conference Proceedings (to be published).

[26] S. Calatroni, E. Garcia-Tabares Valdivieso, A. T. P. Fontenla, M. Taborelli, H. Neupert, M. Himmerlich, P. Chiggiato, D. Bajek, S. Wackerow, and A. Abdolvand, Phys. Rev. Accel. Beams 23, 033101 (2020).

[27] S. Calatroni, M. Arzeo, S. Aull, M. Himmerlich, P. Costa Pinto, W. Vollenberg, B. Di Girolamo, P. Cruikshank, P. Chiggiato, D. Bajek, S. Wackerow, and A. Abdolvand, Phys. Rev. Accel. Beams 22, 063101 (2019).

[28] A. Mathewson, J. P. Bacher, K. Booth, R. Calder, G. Dominichini, A. Grillot, N. Hilleret, D. Latorre, F. Le Normand, and W. Unterlerchner, J. Vac. Sci. Technol. A 7, 77 (1989).

[29] H. J. Halama, AIP Conf. Proc. 199-1, 93 (1990).

[30] I. Collins, P. M. Strubin, O. Gröbner, O. Malyshev, R. Veness, and A. Rossi, LHC Project Report No. 312, CERN, Geneva, 1999.

[31] O. Gröbner, CERN Yellow Report No. 2004-008, 2004, p. 396.

[32] J. Thrower, M. Collings, F. Rutten, and M. McCoustra, Mon. Not. R. Astron. Soc. 394, 1510 (2009).

[33] A. Paldor, G. Toker, Y. Lilach, and M. Asscher, Phys. Chem. Chem. Phys. 12, 6774 (2010).

[34] A. Clemens, L. Hellberg, H. Grönbeck, and D. Chakarov, Phys. Chem. Chem. Phys. 15, 20456 (2013).
[35] T. Suhasaria, J. Thrower, and H. Zacharias, Mon. Not. R. Astron. Soc. 454, 3317 (2015).

[36] L. Spallino, M. Angelucci, R. Larciprete, and R. Cimino, Appl. Phys. Lett. 114, 153103 (2019).

[37] R. E. Honig and H. O. Hook, RCA Rev. 21, 360 (1960).

[38] W. C. Turner, J. Vac. Sci. Technol. A 14, 2026 (1996).

[39] V. Baglin, in 1st LHC Project Workshop, Accelerators and Storage Rings (CERN, Geneva, 2004), p. 275.

[40] R. Larciprete, D. Grosso, M. Commisso, R. Flammini, and R. Cimino, Phys. Rev. ST Accel. Beams 16, 011002 (2013).

[41] R. Cimino, M. Commisso, D. R. Grosso, T. Demma, V. Baglin, R. Flammini, and R. Larciprete, Phys. Rev. Lett. 109, 064801 (2012).

[42] R. Cimino, L. A. Gonzalez, R. Larciprete, A. Di Gaspare, G. Iadarola, and G. Rumolo, Phys. Rev. ST Accel. Beams 18, 051002 (2015).

[43] L. Gonzalez, M. Angelucci, and R. Larciprete, and R. Cimino, AIP Adv. 7, 115203 (2017).

[44] E. Wallén, J. Vac. Sci. Technol. A 14, 2916 (1996).

[45] D.-H. Wei and D. Skelton, and S. Kevan, J. Chem. Phys. 105, 7808 (1996).

[46] J. Ahner, D. Mocuta, R. Ramsier, and J. T. Yates, Jr., J. Chem. Phys. 105, 6553 (1996).

[47] W. Berthold, P. Feulner, and U. Höfer, Chem. Phys. Lett. 358, 502 (2002).

[48] G. A. Kimmel, M. Persson, Z. Dohnalek, and B. D. Kay, J. Phys. Chem. 119, 6776 (2003).

[49] D. P. Engelhart, R. J. Wagner, P. C. Johnsen, and A. M. Wodtke, and T. Schäfer, Phys. Chem. Chem. Phys. 17, 11540 (2015).

[50] A. Potapov, C. Jäger, and T. Henning, Phys. Rev. Lett. 124, 221103 (2020).

[51] J. Cazaux, Y. Bozhko, and N. Hilleret, Phys. Rev. B 71, 035419 (2005).

[52] R. S. Smith, R. A. May, and B. D. Kay, J. Phys. Chem. B 120, 1979 (2016).

[53] T. Suhasaria, J. Thrower, and H. Zacharias, Mon. Not. R. Astron. Soc. 472, 389 (2017).

[54] J. Noble, E. Congiu, F. Dulieu, and H. Fraser, Mon. Not. R. Astron. Soc. 421, 768 (2012).

[55] A. Ferreira and L. Lobo, J. Chem. Thermodyn. 40, 1621 (2008).

[56] V. Baglin, I. Collins, O. Gröbner, C. Grünhagel, and B. Jenninger, Vacuum 67, 421 (2002).

[57] J.-Y. Park, S.-J. Kahng, U. D. Ham, Y. Kuk, K. Miyake, K. Hata, and H. Shigekawa, Phys. Rev. B 60, 16934 (1999).

[58] P. Ayotte, R. S. Smith, K. P. Stevenson, Z. Dohnálek, G. A. Kimmel, and B. D. Kay, J. Geophys. Res. Planets 106, 33387 (2001).

[59] G. Moulard, B. Jenninger, and Y. Saito, Vacuum 60, 43 (2001).

[60] L. Baker, B. Holsclaw, A. E. Baber, H. L. Tierney, E. C. H. Sykes, and A. J. Gellman, J. Phys. Chem. C 114, 18566 (2010). 